\title{
A Novel, Nondestructive, Dried Blood Spot-Based Hematocrit Prediction Method Using Noncontact Diffuse Reflectance Spectroscopy
}

\author{
Sara Capiau, ${ }^{\dagger, \S}$ Leah S. Wilk, ${ }^{\ddagger}, \S$ Maurice C. G. Aalders, ${ }^{\ddagger}$ and Christophe P. Stove ${ }^{* \dagger}$
}

${ }^{\dagger}$ Laboratory of Toxicology, Faculty of Pharmaceutical Sciences, Ghent University, Ottergemsesteenweg 460, 9000 Ghent, Belgium

${ }^{*}$ Department of Biomedical Engineering and Physics, Academic Medical Center, University of Amsterdam, Meibergdreef 9, 1105 AZ Amsterdam, The Netherlands

\section{Supporting Information}
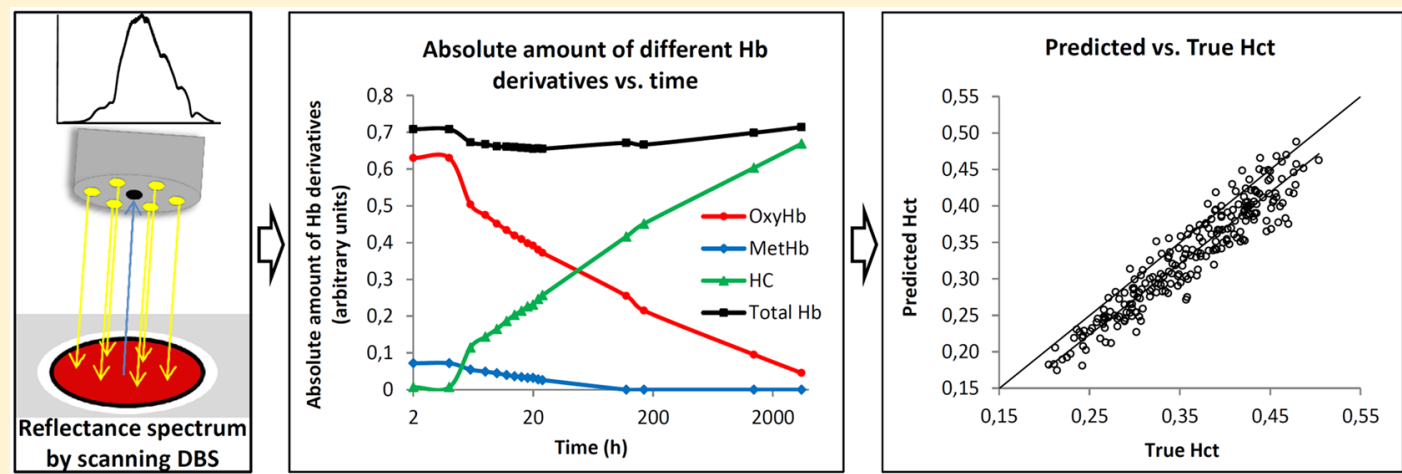

ABSTRACT: Dried blood spot (DBS) sampling is recognized as a valuable alternative sampling strategy both in research and in clinical routine. Although many advantages are associated with DBS sampling, its more widespread use is hampered by several issues, of which the hematocrit effect on DBS-based quantitation remains undoubtedly the most widely discussed one. Previously, we developed a method to derive the approximate hematocrit from a nonvolumetrically applied DBS based on its potassium content. Although this method yielded good results and was straightforward to perform, it was also destructive and required sample preparation. Therefore, we now developed a nondestructive method which allows to predict the hematocrit of a DBS based on its hemoglobin content, measured via noncontact diffuse reflectance spectroscopy. The developed method was thoroughly validated. A linear calibration curve was established after $\log / \log$ transformation. The bias, intraday and interday imprecision of quality controls at three hematocrit levels and at the lower and upper limit of quantitation (0.20 and 0.67 , respectively) were less than $11 \%$. In addition, the influence of storage and the volume spotted was evaluated, as well as DBS homogeneity. Application of the method to venous DBSs prepared from whole blood patient samples $(n=233)$ revealed a good correlation between the actual and the predicted hematocrit. Limits of agreement obtained after Bland and Altman analysis were -0.076 and +0.018 . Incurred sample reanalysis demonstrated good method reproducibility. In conclusion, mere scanning of a DBS suffices to derive its approximate hematocrit, one of the most important variables in DBS analysis.

$\mathrm{O}$ ver the last decades, dried blood spot (DBS) sampling has been increasingly recognized as a valuable alternative sampling strategy in ample domains, ${ }^{1}$ such as newborn screening, ${ }^{2}$ therapeutic drug monitoring, , $^{3,4}$ and toxicology, because of the various advantages that are associated with it. Indeed, DBS sampling is minimally invasive, requires only very small volumes of blood and allows for home-based patient selfsampling. ${ }^{7}$ Nonetheless, DBSs still struggle to be implemented on a routine basis in quantitative bioanalysis. Among the prime causes for this is the so-called hematocrit (Hct) effect, which entails that the Hct of the blood used to prepare a DBS may influence the obtained result. ${ }^{8}$ Indeed, with varying Hct, and hence, varying blood viscosity, blood will spread differently throughout the filter paper, leading to a different volume of blood being analyzed when a fixed-size DBS punch is sampled.
The latter is often preferred over whole DBS analysis after volumetric DBS application to enable simplicity of sample collection, especially when samples need to be collected by the patient himself in a home setting. In addition, the Hct may also influence analyte recovery, matrix effect and DBS homogeneity. ${ }^{9}$ Driven by the impact of this Hct issue on the future of quantitative DBS analysis, ample solutions have already been proposed. These include the analysis of complete fixed-volume DBSs $^{10-14}$ or other dried blood samples, ${ }^{15,16}$ Hct prediction and subsequent Hct effect correction, ${ }^{17,18}$ the use of new

Received: April 5, 2016

Accepted: May 20, 2016

Published: May 20, 2016 
substrates on which the spreading of the blood is Hctindependent, ${ }^{19}$ and the use of in situ-generated dried plasma spots (DPSs). ${ }^{20,21}$ However, all of these solutions have certain limitations or drawbacks, as has been elaborately discussed elsewhere. $8,22-24$

Previously, we developed a method which uses the potassium $\left(\mathrm{K}^{+}\right)$content of a DBS extract to predict its Hct. ${ }^{17}$ Although this method yielded excellent results when applied to real patient samples and was able to adequately correct for the Hct effect using caffeine and paraxanthine as model compounds, ${ }^{18}$ it suffered from some practical drawbacks. Indeed, part of the DBS needed to be sacrificed for the $\mathrm{K}^{+}$analysis, which also required an additional sample preparation. Therefore, we set out to develop a noncontact method to predict the Hct of DBSs, since such a method would preserve the entire DBS sample and exclude the need for sample preparation, which in turn could facilitate integration in existing DBS analyzers. More particularly, it was our goal to develop a noncontact method that allows to predict the Hct of a DBS, based on its total $\mathrm{Hb}$ content.

Since previous experiments performed at our laboratory showed that $\mathrm{Hb}$, upon aging of DBSs, is converted from oxyhemoglobin $(\mathrm{OxyHb})$ to methemoglobin $(\mathrm{MetHb}){ }^{8}$ which is also accompanied by spectral changes (cfr. the change in color of a DBS upon aging), both $\mathrm{Hb}$ forms need to be taken into account to allow spectrometry-based $\mathrm{Hb}$ quantitation. However, even when doing so, the $\mathrm{Hb}$ sum proved to be unstable in function of time. Interestingly, Bremmer et al. demonstrated that in aging blood stains $\mathrm{Hb}$ is originally present as $\mathrm{OxyHb}$, which is then oxidized to $\mathrm{MetHb}$ and further denatured to hemichrome (HC). Therefore, we hypothesized that if a similar process would occur in DBSs and no other major $\mathrm{Hb}$ derivatives or metabolites were formed upon aging, the sum of $\mathrm{OxyHb}, \mathrm{MetHb}$ and $\mathrm{HC}$ (i.e., "total $\mathrm{Hb}$ ") would remain constant and could be used as a marker of Hct. As a basis for the development of the noncontact method we used the work of Bremmer et al. and Edelman et al., who used noncontact diffuse reflectance spectroscopy to determine the relative abundance of $\mathrm{Oxy}-\mathrm{Hb}, \mathrm{MetHb}$ and $\mathrm{HC}$ in dried blood stains at crime scenes for age estimation purposes. ${ }^{25,26}$ For the Hct prediction of DBSs, however, these three $\mathrm{Hb}$ derivatives had to be determined quantitatively, rather than qualitatively, which required alteration and optimization of the method.

Total $\mathrm{Hb}$ has been previously determined colorimetrically in DBS extracts either "as is" ${ }^{8}$ or after transformation of $\mathrm{Hb}$ into its cyano-methemoglobin derivative via extraction of the DBS in Drabkin's reagent. ${ }^{27-29}$ However, in each case the age of the DBSs influenced the obtained results. Others have used the total area under the curve of all $\mathrm{Hb}$ variants determined via HPLC-UV during hemoglobinopathy screening as a measure of total $\mathrm{Hb}$ and $\mathrm{Hct}$, albeit only semiquantitative results could be achieved. ${ }^{30}$ Furthermore, LC-MS/MS has been successfully employed to determine total $\mathrm{Hb}$ by measuring a proteospecific peptide after tryptic digestion of a DBS extract. ${ }^{31}$ Unfortunately, this method is not suitable for Hct prediction of DBSs, since in many cases the Hct prediction would be more complicated than the analysis of the actual target analyte. Additionally, Miller et al. tried to utilize the $\mathrm{Hb}$ bands at 540 and/or $570 \mathrm{~nm}$, measured with noncontact diffuse reflectance spectroscopy, as a measure of a DBS ' Hct. ${ }^{32}$ Although these authors did not find a correlation between the Hct and the reflectance at these $\mathrm{Hb}$-specific wavelengths, they did find a correlation with the background scattering measured at 980 nm. The same optical technique has also been employed by Cecchi et al. to measure total $\mathrm{Hb}$ after transformation of $\mathrm{Hb}$ into its isothiocyano-methemoglobin derivative. ${ }^{33}$ To that end, derivatizing reagents were preimpregnated in a nylon-based filter paper used to collect DBSs. Another noncontact method to determine total $\mathrm{Hb}$ was published by Yang et al., which encompassed mixing Drabkin's reagent and blood before deposition of the sample onto the filter paper and using the color intensity determined on a digitized image of the DBS as a measure of total $\mathrm{Hb}^{34}$ However, such an approach would not be feasible in the context of home-sampling.

In this Article, we describe a method that allows to predict the Hct of a regular, nonvolumetrically applied DBS, based on its $\mathrm{Hb}$ content using noncontact diffuse reflectance spectroscopy. In contrast to other published DBS-based total $\mathrm{Hb}$ analyses, this method is quantitative, nondestructive, applicable on both fresh and old DBS, does not require any sample preparation nor filter paper pretreatment, and allows to predict the Hct of a DBS. This method was thoroughly validated, yielding acceptable accuracy and precision, and was applied to a diverse patient population, yielding satisfactory results.

\section{EXPERIMENTAL SECTION}

Preparation of DBS Samples. Venous blood was collected from consenting healthy volunteers in blood collection tubes with lithium heparin (Li-heparin) as anticoagulant (Venosafe, VF-109SHL, Terumo, Leuven, Belgium). DBSs were prepared at the day of blood collection by depositing $25 \mu \mathrm{L}$ of blood onto Whatman 903 filter paper (GE Healthcare, Dassel, Germany), unless mentioned otherwise. Blood spots were always allowed to dry at ambient conditions for at least $2 \mathrm{~h}$. The obtained DBSs were either analyzed immediately after drying or were stored in zip-locked plastic bags in the presence of a desiccant until analysis (Minipax, Sigma-Aldrich, Diegem, Belgium). Blood samples with different Hct were prepared by centrifuging an aliquot of venous whole blood in $2 \mathrm{~mL}$ safe-lock tubes in an Eppendorf 5417R centrifuge (Eppendorf, Hamburg, Germany) for $5 \mathrm{~min}$ at $1000 \mathrm{~g}$, followed by the removal or addition of a suitable amount of plasma. For evaluating the influence of the presence of different anticoagulants on the measured reflectance, venous blood from a healthy volunteer was collected in blood collection tubes with either Li-heparin, sodium fluoride $(\mathrm{NaF})$ /oxalate (Venosafe, VF-052SFX, Terumo), ethylenediaminetetraacetic acid dipotassium ( $\mathrm{K}_{2}$ EDTA) or citrate as anticoagulant and in a blood collection tube without any anticoagulant (all from BD Vacutainer, Becket Dickinson B.V., Breda, The Netherlands). The DBSs without anticoagulant were prepared within $1 \mathrm{~min}$ after blood collection. Patient samples were prepared similarly to the ones from healthy volunteers and were collected in Li-heparin (Venosafe, VF-106SAHL and VF-054SAHL, Terumo) or $\mathrm{K}_{2}$ EDTA-containing blood collection tubes (Venosafe, VF054SDK, Terumo).

Analysis. Hct measurements on venous whole blood were performed using a Sysmex XE-5000 hematology analyzer (Sysmex Corporation, Kobe, Japan) and are expressed as L/L throughout the manuscript. All Hct measurements during method development and validation were performed at the Laboratory of General Clinical Chemistry (LAKC) at the Academic Medical Center (AMC), Amsterdam, while the Hct measurements of the patient samples were performed at the Laboratory of Clinical Biology at Ghent University Hospital. The analyzer at Ghent University Hospital was also used for the 
mean corpuscular hemoglobin concentration (MCHC) analysis of the patient samples. Additionally, the hemolytic, lipemic and icteric index of the patient samples were determined using a Cobas 8000 analyzer at Ghent University Hospital.

Noncontact diffuse reflectance spectroscopy was employed to determine the total $\mathrm{Hb}$ content and $\mathrm{Hct}(\mathrm{L} / \mathrm{L})$ of the DBSs. The setup that was used to acquire the spectral data (depicted in Figures 1 and S-1) comprised a 10W tungsten-halogen light



Figure 1. Schematic representation of the used setup.

source (AvaLight-HAL, Avantes, Appeldoorn, The Netherlands), a spectrometer (USB4000, Ocean Optics, Duiven, The Netherlands) and a noncontact fiber-probe (QR400-7-UV/BX, Ocean Optics, Duiven, The Netherlands). The fiber-probe consisted of a collection fiber encircled by six delivery fibers. All fibers had a core diameter of $400 \mu \mathrm{m}$. The probe tip was placed $1.3 \mathrm{~cm}$ above the sample and set at a slight angle to avoid specular reflection. Moreover, a neutral density filter with an optical density of 0.4 was inserted between the light source and the delivery fibers to prevent detector saturation. In this setup, the light source output was guided toward the sample surface via the six delivery fibers, illuminating a $5.9 \mathrm{~mm}$-diameter spot, which corresponds approximately to a traditional $6 \mathrm{~mm}$-punch. Light reflected by the DBS was guided to the spectrometer by the central collection fiber. The spectrometer recorded the wavelength dependence of the reflected light intensity (reflection spectrum) between 354 and $1042 \mathrm{~nm}$ using the SpectraSuite software (Ocean Optics, Duiven, The Netherlands). In addition to the recording of a reflection spectrum of each DBS, also the reflection spectrum of a white reference was recorded. The integration time was set at $20 \mathrm{~ms}$ and 20 spectra were averaged for one measurement. All recorded spectra were corrected for the electrical dark noise of the detector.

Spectral Data Analysis. All data analysis was carried out using custom-made scripts written in MATLAB R2012a (The Mathworks Inc., Natick, MA, USA). Wavelength-dependent reflectance values $R(\lambda)$ were obtained from the measured spectra using the following formula (eq 1):

$$
R(\lambda)=\frac{I(\lambda)}{I_{\text {white }}(\lambda)}
$$

Here, $\lambda$ corresponds to the wavelength of the light. Further, $I(\lambda)$ refers to the light intensity reflected by a DBS, while $I_{\text {white }}(\lambda)$ denotes the light intensity reflected by the white reference. Applying eq 1 to the data served as a normalization step, normalizing the light intensity reflected by the DBSs to the light intensity reflected by the nonlight-absorbing white reflectance standard. $R(\lambda)$ was then compared to a onedimensional light-transport model for multilayered samples. ${ }^{35}$ This light-transport model describes the wavelength-dependent reflectance of a DBS as a combination of light absorption and light scattering and takes into account the optical properties of both the blood and the blank filter paper. In this model the influence of the blank filter paper is described by its measured wavelength-dependent reflectance and the wavelength-dependent optical properties of the blood are described by an absorption coefficient and a scattering coefficient. Light absorption in the DBSs is mainly attributed to three hemoglobin derivatives which are assumed to be present, namely, $\mathrm{OxyHb}$, MetHb, and HC. The overall absorption coefficient of the blood is therefore modeled as a linear combination of the individual absorption coefficients of the three $\mathrm{Hb}$ derivatives. Reference curves for these three absorption coefficients were taken from Bremmer et al. ${ }^{25}$ For a given DBS, the present amounts of the $\mathrm{Hb}$ derivatives were calculated by fitting the output of the light-transport model to the measured $R(\lambda)$ in the spectral range of 500-700 nm, employing a nonlinear least-squares fitting algorithm. The range of 500-700 $\mathrm{nm}$ was used in the fitting procedure, since during method optimization this proved to yield the best fit between the measured spectra and the reference spectra. The least-squares fitting algorithm assigned a value with arbitrary units to each of the $\mathrm{Hb}$ derivatives and the sum of those values was then used as a measure of total $\mathrm{Hb}$ and Hct. To further clarify the spectral data analysis process, a flowchart was included in Figure S-2.

Validation. To choose a calibration model, we set up six 7point calibration curves in DBSs, prepared from blood with a Hct of $0.20,0.27,0.35,0.41,0.49,0.57$, and 0.66 . All calibrators were generated from the blood of a single donor. Unweighted and weighted $\left(1 / x, 1 / x^{2}, 1 / y, 1 / y^{2}\right)$ linear regression models, a second order regression model, a power regression model, as well as a linear regression model after transformation of the data (logaritmic or square root) were compared. The choice of the calibration model was based upon the sum of the absolute percent relative errors (\% RE) and the distribution of the RE in function of Hct. ${ }^{36}$ Linearity was evaluated using an F-test, acceptance of a linear model was based upon the backcalculated values of all calibrators. A Levene's test for equality of variances was performed to assess homoscedasticity.

Accuracy (\% bias) and precision (\% RSD, relative standard deviation) were determined based on six 7-point calibration curves in DBSs, prepared from blood with an approximate Hct of $0.20,0.27,0.35,0.42,0.50,0.57$, and 0.65 . On each of three different days two calibration curves were set up. Along with every calibration curve, three DBS quality controls (QCs) with an approximate Hct of $0.20,0.42$, and 0.65 were prepared from blood of the same donor. Furthermore, additional QCs were prepared from five other blood sources. In addition, six 7-point calibration curves were also prepared from the blood of a second donor to exclude that the obtained results would be donor-dependent. An overview of all calibrators and QCs is given in Table S-1. As it is not possible to prepare QCs with exactly the same Hct for each measurement series, these slight differences in true Hct had to be taken into account for the determination of precision. This was done by normalizing the calculated Hct values to the average true Hct value of a QC (Table S-2A and S2-B).

Hct predictions based on measurements at the center of the DBS were compared with those obtained from measurements at a more peripheral location, avoiding the very edge of the sample $(n=6)$. This experiment was performed on $50 \mu \mathrm{L}$ DBSs to allow sufficient difference in location between a central 
and peripheral measurement. The impact of the blood volume spotted was evaluated in DBSs prepared by applying 15, 20, 25, or $50 \mu \mathrm{L}$ of blood on filter paper $(n=6)$. Both the impact of the measuring location and of the applied blood volume were evaluated at three Hct levels (approximately 0.20, 0.42, and $0.65)$.

The effect of storage of the DBSs on the Hct prediction was evaluated by comparing the result from freshly prepared DBSs (dried for $2 \mathrm{~h}$; three Hct levels, six replicates) with results obtained after storage at room temperature for 1 day, 5 days, 1 week, 2 months, and 5 months. In addition, the influence of storage at $60{ }^{\circ} \mathrm{C}$ for 1,2 or 3 days on the predicted Hct was evaluated as well. Results were expressed as the average $(n=6)$ \pm standard deviation (SD). Thanks to the nondestructive nature of the DBS analysis, the fresh spots could be reanalyzed at later time points. At every time point a fresh calibration curve (dried for $2 \mathrm{~h}$ ) was prepared.

Application. The developed method was applied on reallife clinical samples displaying a wide range of Hct values (0.205-0.504). The samples had already undergone or were destined for routine clinical chemistry and/or hematology analysis and were collected in either Li-heparin tubes or in $\mathrm{K}_{2}$ EDTA tubes. Permission to use these samples was obtained from the Ethics Committee of Ghent University Hospital (project number 2015/0931). A first set of samples encompassed $57 \mathrm{Li}$-heparin whole blood samples that were used to prepare DBSs upon arrival at the clinical laboratory by pipetting $25 \mu \mathrm{L}$ of Li-heparin anticoagulated blood onto filter paper. We also prepared DBSs of the corresponding $\mathrm{K}_{2}$ EDTA whole blood samples of these patients. A second set of samples encompassed $250 \mathrm{~K}_{2}$ EDTA left-over samples from which we prepared DBSs on the same day of blood collection. Samples for which the routinely determined Hct or $\mathrm{MCHC}$ value were not available were excluded from further data analysis, as will be discussed in more detail below. This resulted in a final data set of 55 Li-heparin-containing samples with corresponding $\mathrm{K}_{2}$ EDTA samples and 233 " $\mathrm{K}_{2}$ EDTA-only" samples. The DBS were between 5 and 7 days old at the time of analysis.

Incurred sample reanalysis (ISR) - with 3 days between the reanalysis and the original analysis-was performed on the second set of patient DBSs $(n=233)$. At least two-thirds of the repeated measurements should meet the acceptance criterion, that is, lie within the limits of $\pm 20 \%$ of the mean of the original and the corresponding reanalysis result. ${ }^{37}$

Data Analysis. Statistical analysis of the data was done using Medcalc Statistical Software, version 14.12.0 (MedCalc Software bvba, Ostend, Belgium, http://www.medcalc.org, 2014), SPSS statistics for Windows version 22.0 (IBM Corp, Armonk, NY, USA, 2013) and Microsoft Excel 2010. For the determination of intra- and interday imprecision, a model II ANOVA was used to allow analysis of the components of variation. Using this ANOVA approach, the value of the interday imprecision was equated to the intraday imprecision, whenever the latter would exceed the former. A paired $t$ test $(\alpha$ $=0.05 ; 95 \% \mathrm{CI}$, confidence interval) was carried out to evaluate the effect of the measurement location on the predicted Hct. To evaluate the influence of different volumes of blood spotted, a one-way ANOVA $(\alpha=0.05)$ with Bonferroni posthoc test was employed. Medcalc was used to perform a Levene's test for the evaluation of homoscedasticity. Furthermore, the same software was employed to calculate Pearson correlation coefficients, to generate Mountain plots and to perform Passing and Bablok regression analysis on the patient data. The corresponding Bland and Altman plots were generated in Excel 2010. Furthermore, a paired $t$ test was employed to evaluate whether a statistically significant difference existed between the predicted Hct determined on Li-heparin-containing DBSs and the predicted Hct determined on $\mathrm{K}_{2}$ EDTA-containing DBSs. The presence of outliers was always evaluated using a Grubbs test.

\section{RESULTS AND DISCUSSION}

Method Development. Optimization of Setup. To obtain reproducible reflectance spectra, it was of utmost importance to keep the distance between the probe and the sample constant. To achieve this, the setup needed to be fixed and the filter paper (in sheets, spotted with multiple DBSs) needed to be kept flat. To that end, a plate was positioned on top of the filter paper and pressed down with weights. This plate had a $1 \mathrm{~cm}$ hole in the center (i.e., slightly larger than the DBS that needed to be analyzed) through which the measurement could be performed. Importantly, when using DBS cards instead of sheets of filter paper, the "waviness" of the substrate after DBS application and drying is much less of a problem, since the former are much more rigid in nature. Evidently, although the method could potentially be applied on either DBS cards or sheets of filter paper, it is important to prepare calibrators and patient samples on the same substrate and to record the respective spectra with exactly the same setup and hence, with the same fixed distance between DBS and probe.

To optimize the distance between the probe and the sample, three different distances (i.e., 0.7, 1.3, and $1.7 \mathrm{~cm}$ ) were compared, yielding illuminated areas of respectively approximately 3,6 , and $8 \mathrm{~mm}$. The distance of $0.7 \mathrm{~cm}$ resulted in a larger variability in the obtained spectra compared to the two larger distances (data not shown), likely because of some inhomogeneity in the DBSs, which we could also observe via light microscopy (Figure S-3). Since increasing the distance from 1.3 to $1.7 \mathrm{~cm}$ did not lead to a further improvement (i.e., a further reduction in variability), the distance of $1.3 \mathrm{~cm}$ was selected for the rest of the experiments. The resulting illumination diameter of $5.9 \mathrm{~mm}$ is acceptable, since DBSs generated from a finger prick will typically have a larger diameter. ${ }^{7,38,39}$

Additionally, the filter paper was positioned on a black matt surface to ensure that light going through the filter paper was absorbed by the background and not reflected toward the collection fiber. Indeed, with varying Hct, and hence variable amounts of chromophores in a DBS, variable amounts of light may penetrate the sample and get reflected by, for example, a white background, which would lead to a variable, Hctdependent contribution of the background to the measured reflectance.

Selection of the Used Anticoagulant. A procedure aimed at measuring Hct in DBSs should be applicable to nonanticoagulated blood, since this is the type of blood that eventually will be analyzed (e.g., obtained from a finger prick in the context of home-based self-sampling). However, because of practical reasons, it is impossible to perform a validation or even to set up calibration curves in nonanticoagulated blood. We therefore compared at an early stage the reflectance spectra of DBSs prepared from blood without anticoagulant with the reflectance spectra of DBSs from blood that was anticoagulated with $\mathrm{Li}$-heparin, $\mathrm{K}_{2} \mathrm{EDTA}$, citrate, or $\mathrm{NaF}$ /oxalate. The reflectance spectra of $\mathrm{K}_{2}$ EDTA and Li-heparin-containing DBSs were nearly identical to the spectra of nonanticoagulated 
DBSs (Figure S-4A and S-4B). The spectra of the citratecontaining DBSs on the other hand had a similar shape as the spectra from the nonanticoagulated DBSs but showed a markedly higher reflectance. Furthermore, the spectra of the $\mathrm{NaF}$ /oxalate-containing DBSs showed a slightly deviating shape. Therefore, only Li-heparin and $\mathrm{K}_{2}$ EDTA were deemed suitable anticoagulants to develop the noncontact Hct prediction method. At this stage, we wished to preserve the possibility to also analyze the DBS samples with the existing $\mathrm{K}^{+}$based method for Hct prediction. Therefore, we opted for Liheparin anticoagulated blood to perform the validation.

Method Validation. A power regression model (and the derived linear regression model after $\log / \log$ transformation) yielded the best calibration model. Furthermore, although data were originally heteroscedastic $(p=0.005)$, they became homoscedastic after $\log / \log$ transformation $(p=0.429)$. Although an F-test performed on the logarithmically transformed data showed that the calibration model was statistically nonlinear, a linear model could be accepted based upon the backcalculated values of all calibrators, which never deviated more than $15 \%$ (range $=-11.57 \%$ to $10.48 \%$ ). The lower and upper limit of quantification (LLOQ and ULOQ) were arbitrarily set at the average of the lowest, respectively, the highest calibrator. For donor 1 the LLOQ and ULOQ corresponded to 0.20 and 0.67 , while for donor 2 this was 0.20 and 0.65 , respectively. The accuracy, intra- and interday precision for LLOQ and ULOQ were determined on the backcalculated values and always met the predetermined acceptance criteria (i.e., $\leq 20 \%$ bias and imprecision for LLOQ and $\leq 15 \%$ for ULOQ) (Table 1A). Also for the three QC levels \% bias and $\%$ RSD did not exceed $11 \%$ (Table 1B). As described above, this experiment was also carried out using blood of a second donor, yielding very similar results (see Table S-3). Also when the QCs of donor 2 were inserted in the calibration curve of donor 1 (or vice versa) the results always complied with the acceptance criteria (Tables 1C and S-3). Moreover, QCs prepared from four additional blood sources (donors 3-6) were fitted into the calibration curves of donor 1 and donor 2, again leading to acceptable results (Tables 1C and S-3).

As mentioned above, the reflectance spectrum of a DBS changes drastically upon aging because the relative amounts of the different $\mathrm{Hb}$ derivatives change throughout time (Figure 2A and $2 \mathrm{~B}$ ). It was therefore of utmost importance to evaluate the influence of storage time on the predicted Hct. Long-term stability at room temperature did not seem to pose a problem for up to at least 5 months, as, with a single exception, the observed differences never exceeded $15 \%$ at all measured time points and at every Hct level (Figure 2C). Also short-term storage (up to 3 days) at elevated temperatures $\left(60^{\circ} \mathrm{C}\right)$ did not seem to affect the obtained results, as can be seen in Figure 2D.

Although one-way ANOVA showed a statistically significant difference in predicted Hct between DBSs prepared from 15, 20,25 , and $50 \mu \mathrm{L}$ of blood at all the Hct levels tested ( $p<$ 0.05 ), posthoc analysis revealed that there was no statistical difference for any of the applied volumes when compared to the $25 \mu \mathrm{L}$ reference volume. Moreover, these differences were sufficiently small to not pose a problem in practice (i.e., $\leq 8 \%$ compared to the reference of $25 \mu \mathrm{L}$ ) (Figure 3A). These differences are likely the result of a different degree of saturation of the filter paper, with higher sample volumes leading to a slightly higher saturation of the filter paper when compared to smaller volumes. This way, a smaller volume of blood will be present in a fixed-size DBS punch when a small
Table 1. Overview of the Data for Accuracy and Precision ( $n$ $=6$ ) for Donor $1^{a}$

\begin{tabular}{|c|c|c|c|}
\hline & $\begin{array}{l}\text { accuracy } \\
\text { (\% bias) }\end{array}$ & $\begin{array}{l}\text { intraday precision } \\
\text { (\% RSD) }\end{array}$ & $\begin{array}{l}\text { interday precision } \\
\text { (\% RSD) }\end{array}$ \\
\hline \multicolumn{4}{|c|}{ (A) } \\
\hline LLOQ & 2.41 & 2.90 & 3.97 \\
\hline \multicolumn{4}{|c|}{ (B) } \\
\hline QC LOW & 0.896 & 10.7 & 10.7 \\
\hline QC MID & -2.97 & 2.10 & 3.08 \\
\hline QC HIGH & \multicolumn{2}{|r|}{ (C) } & 4.63 \\
\hline $\begin{array}{l}\text { QC LOW } \\
\text { (donor 2) }\end{array}$ & -1.81 & 2.30 & 5.65 \\
\hline $\begin{array}{l}\text { QC MID } \\
\text { (donor 2) }\end{array}$ & 0.630 & 5.56 & 6.91 \\
\hline $\begin{array}{l}\text { QC HIGH } \\
\text { (donor 2) }\end{array}$ & 0.857 & 7.38 & 7.38 \\
\hline $\begin{array}{l}\text { QC LOW } \\
\text { (donor 3) }\end{array}$ & 3.42 & 5.12 & 6.80 \\
\hline $\begin{array}{l}\text { QC MID } \\
\text { (donor 3) }\end{array}$ & 5.48 & 2.67 & 3.67 \\
\hline $\begin{array}{l}\text { QC HIGH } \\
\text { (donor 3) }\end{array}$ & 2.65 & 3.89 & 5.36 \\
\hline $\begin{array}{l}\text { QC LOW } \\
\text { (donor 4) }\end{array}$ & 1.62 & 6.42 & 6.42 \\
\hline $\begin{array}{l}\text { QC MID } \\
\text { (donor 4) }\end{array}$ & 0.629 & 7.68 & 8.38 \\
\hline $\begin{array}{l}\text { QC HIGH } \\
\text { (donor 4) }\end{array}$ & -2.11 & 5.67 & 5.67 \\
\hline $\begin{array}{l}\text { QC LOW } \\
\text { (donor 5) }\end{array}$ & 4.01 & 7.77 & 7.77 \\
\hline $\begin{array}{l}\text { QC MID } \\
\text { (donor 5) }\end{array}$ & -2.82 & 6.52 & 6.52 \\
\hline $\begin{array}{l}\text { QC HIGH } \\
\text { (donor 5) }\end{array}$ & 0.927 & 3.00 & 3.00 \\
\hline $\begin{array}{l}\text { QC LOW } \\
\text { (donor 6) }\end{array}$ & 5.13 & 5.45 & 6.15 \\
\hline $\begin{array}{l}\text { QC MID } \\
\text { (donor 6) }\end{array}$ & 4.92 & 11.8 & 11.8 \\
\hline QC HIGH & 4.34 & 10.8 & 10.8 \\
\hline
\end{tabular}

(donor 6)

${ }^{a}$ Sections A and B, respectively, give the data obtained for the LOQs (LLOQ and ULOQ) and QCs, prepared from blood of the same donor as the one in which the calibration curves were prepared. Section C gives the data for QCs prepared from blood of five other donors than the one in which the calibration curves were prepared.

volume of blood is deposited on the filter paper, whereas a larger volume of blood will be present in a fixed-size DBS punch when a larger volume of blood is deposited on the filter paper. This is a known phenomenon for DBSs, as we described before. $^{38,40}$

Although a marginally statistically significant difference ( $p=$ 0.04 ) could be observed between measurements performed in the center of DBSs of low Hct $(\mathrm{Hct}=0.19)$ compared to measurements performed at a more peripheral location of the same DBSs, this difference is not of practical relevance (i.e., $\leq$ $4 \%$ ), as can be clearly seen in Figure 3B. Furthermore, for the measurements at both mid $(\mathrm{Hct}=0.41)$ and high $(\mathrm{Hct}=0.65)$ Hct neither a statistically significant $(p=0.07$ and 0.15 , respectively) nor a practically relevant difference was observed.

Method Application. The newly developed noncontact method was applied to patient samples with a wide Hct range (0.205-0.504). The first set of samples included $55 \mathrm{Li}$-heparin DBSs and their corresponding $\mathrm{K}_{2}$ EDTA DBSs. The true Hct of the patient samples was routinely determined on $\mathrm{K}_{2}$ EDTA 


\section{A Reflectance spectra of a DBS upon ageing}

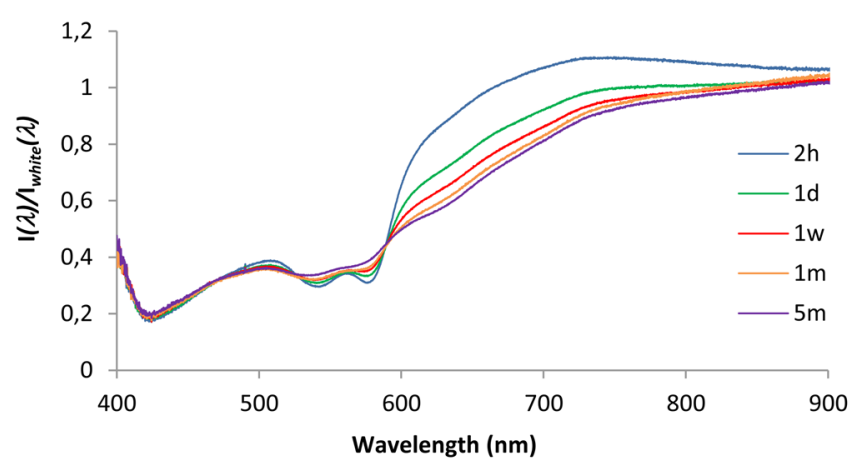

C

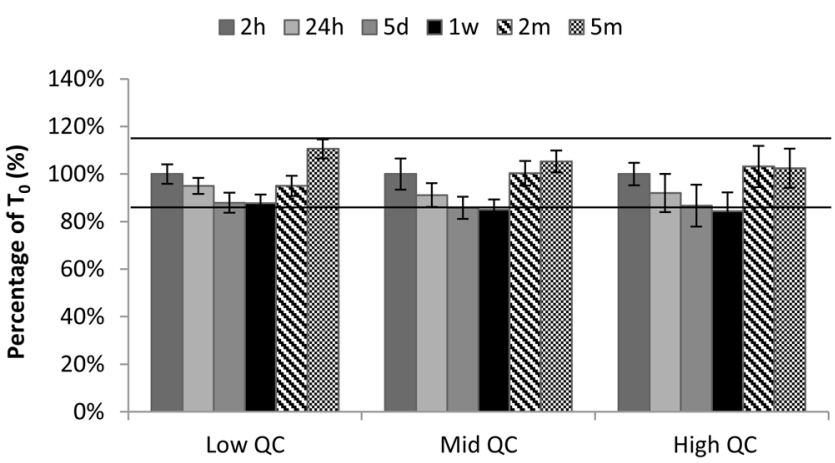

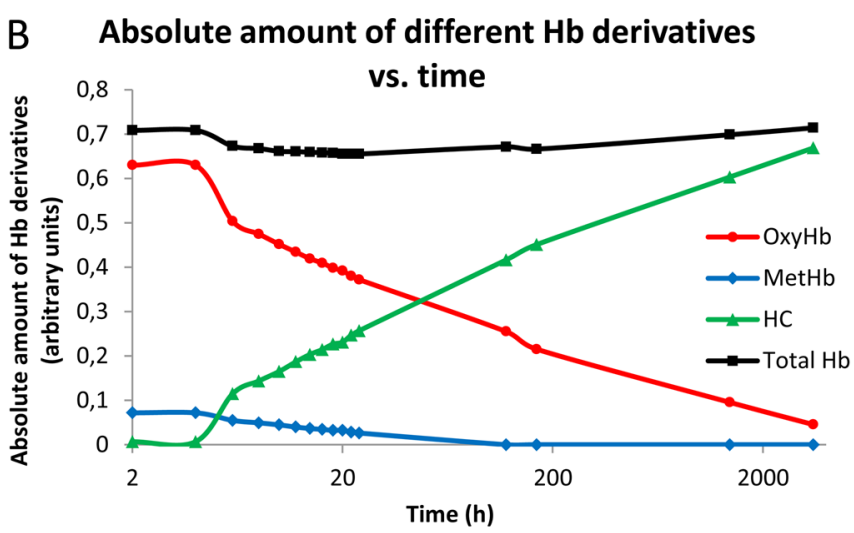

D

Stability at elevated temperatures

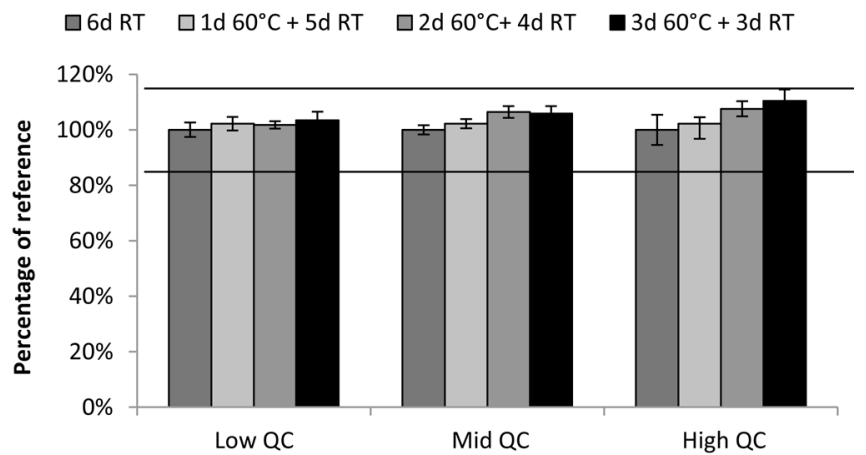

Figure 2. (A) The reflectance spectra of a DBS recorded after $2 \mathrm{~h}, 1$ day, 1 week, 1 month. and 5 months. The DBS which was analyzed had an approximate Hct of 0.20 . (B) The amounts of OxyHb, MetHb, and HC present in DBSs up to five months. The DBSs which were analyzed had an approximate Hct of 0.20 . Note the logaritmic scale of the $x$-axis. (C) The influence of storage at room temperature for up to five months on estimated Hct, at three Hct levels. At each Hct level, the average estimated Hct value at $T_{0}$ was used as a reference value. (D) The influence of storage at elevated temperatures $\left(60^{\circ} \mathrm{C}\right)$ for up to 3 days on estimated Hct, at three Hct levels. At each Hct level, the corresponding DBSs stored under ambient conditions were used as a reference. The horizontal lines indicate the $15 \%$ acceptance levels. For panel $\mathrm{B}, \mathrm{C}$ and $\mathrm{D}$ average values $(n=$ 6) are depicted. In panels $\mathrm{C}$ and $\mathrm{D}$, the error bars indicate SD.

\section{A}

$\square 15 \mu \mathrm{L} \quad \square 20 \mu \mathrm{L} \quad \square 25 \mu \mathrm{L} \quad \square 50 \mu \mathrm{L}$



B


Figure 3. (A) Volume effect: Influence of the volume used to generate DBSs on the estimated Hct, at three Hct levels. Per Hct level the $25 \mu \mathrm{L}$ DBSs were taken as the reference. (B) Volcano effect: Influence of the measurement localization on the estimated Hct, at three Hct levels. Per Hct level the measurements at a central localization were taken as a reference. Depicted in each graph are the averages $(n=6) \pm$ SD. Results differing significantly $(p<0.05)$ from the reference have been indicated with an asterisk.

anticoagulated whole blood, while the noncontact method was employed to predict the Hct of the DBSs. A good correlation ( $r$ $=0.93$ ) was observed between the predicted Hct of the Liheparin DBSs and the true Hct. Nonetheless, Passing and Bablok regression analysis revealed a statistically significant difference. The $95 \%$ CIs of the slope and the intercept were 0.76 to 0.92 and -0.011 to 0.031 , respectively, indicating the presence of a proportional error ( 1 is not included in the CI of the slope) but not of a systemic error ( 0 is included in the CI of the intercept). When the differences between the predicted and the true Hct were plotted versus the true Hct, an average bias of -0.031 could be observed (with a range of -0.069 to 0.014 ). However, importantly, aside from two exceptions which deviated $-21.0 \%$ and $-21.1 \%$, respectively, all predicted Hct values were within $\pm 20 \%$ of the true Hct (Figure S-5), indicating that the noncontact method is fit for purpose (i.e., an approximate prediction of the Hct of a DBS). 

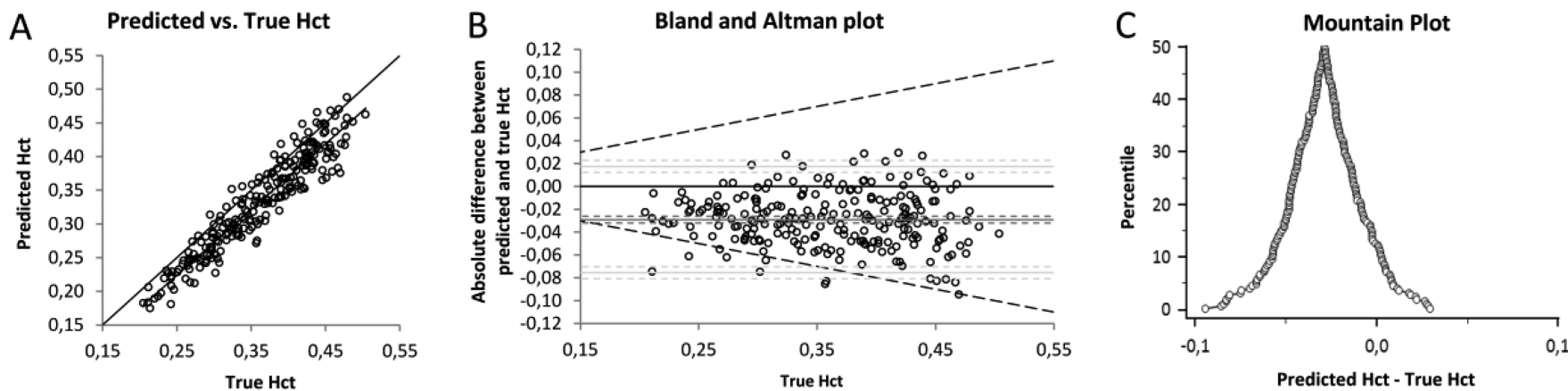

Figure 4. (A) Comparison of predicted and true patient Hct values and corresponding regression line. Also depicted is the line of equality. (B) Bland and Altman comparison of predicted and true Hct. Depicted are the mean difference, the upper and lower LoAs (all with their respective 95\% CIs) and the $20 \%$ acceptance criteria. (C) Mountain plot depicting the distribution of the differences between the predicted and the true Hct. In all graphs, the true Hct refers to the Hct determined on EDTA-anticoagulated whole blood.

Next, the predicted Hct determined on the Li-heparin DBSs was compared with the predicted Hct determined on the corresponding $\mathrm{K}_{2}$ EDTA DBSs. The Pearson correlation coefficient was 0.95 , implying a good correlation between results obtained from Li-heparin and $\mathrm{K}_{2}$ EDTA DBSs. In addition, no statistically significant difference was found between both matrices after applying a paired $t$ test $(p=$ $0.19)$. Therefore, it was concluded that the noncontact method could be applied on both Li-heparin and $\mathrm{K}_{2}$ EDTA DBSs.

In a next step, the method was applied to a second, independent set of $233 \mathrm{~K}_{2}$ EDTA-containing DBSs. Again, a good correlation $(r=0.95)$ was observed between the predicted and true Hct (Figure 4A). Yet, Passing and Bablok regression analysis demonstrated that a slight systemic error was present since the $95 \%$ CI of the intercept did not contain $0(-0.056$ to $-0.022)$, whereas no proportional error could be detected as 1 was included in the $95 \% \mathrm{CI}$ of the slope (0.98 to 1.07 ). Furthermore, a Mountain plot (Figure 4C) depicting the distribution of the differences between the predicted and true Hct, showed a symmetrical distribution around a bias of approximately -0.029 . This can also be observed in the Bland and Altman plot (Figure 4B), showing a mean difference of -0.029 (95\% CI $=-0.032$ to -0.026$)$ and limits of agreement (LoAs) of -0.076 ( $95 \% \mathrm{CI}=-0.081$ to -0.070 ) and 0.018 ( $95 \% \mathrm{CI}=0.012$ to 0.023 ). These LoAs are similar to the ones that we obtained for our $\mathrm{K}^{+}$-based method for Hct prediction which has already proven to successfully alleviate Hct bias in the quantitative DBS-based analysis of caffeine and paraxanhthine. ${ }^{17,18}$ Hence, these LoAs (i.e., the degree of error) were considered acceptable for the intended purpose. Importantly, 95\% of the predicted Hct values were within $20 \%$ of the corresponding true Hct.

We identified several factors that may have contributed to the observed slight negative bias. First, the "true" Hct of the patient samples was determined on $\mathrm{K}_{2}$ EDTA anticoagulated whole blood, while the "true" Hct of the calibrators was routinely determined on Li-heparin anticoagulated whole blood. The latter gives a slightly lower hematocrit than the former, on average. ${ }^{17}$ Second, because of logistical reasons, all Hct measurements on patient whole blood were performed in Ghent University Hospital, whereas the Hct measurements on the whole blood used to prepare the calibrators that were used to analyze the patient samples were performed in the AMC Amsterdam. Small differences may have been present between the results obtained in both laboratories. Furthermore, a clear influence of the patient's MCHC value on the predicted Hct was observed. The MCHC is a measure of the concentration of $\mathrm{Hb}$ in a given number of packed red blood cells and is calculated by dividing the $\mathrm{Hb}$ content of a sample by the Hct. We observed that the lower the patients' MCHC values were, the more pronounced the underestimation of their Hct values was on average (Figure S-6). In addition, the influence of the hemolytic, lipemic, and icteric indexes on the predicted Hct was evaluated. However, none of these parameters seemed to have a noticeable influence on the obtained results (Figure S-7).

Incurred sample reanalysis performed on the second set of patient samples $(n=233)$ revealed good method reproducibility. After the removal of one outlier (54\%; confirmed with a Grubbs test, $p<0.05$ ) all data points were within the acceptance criterion of $\pm 20 \%$ ( $95 \%$ were within $\pm 8 \%$ ).

\section{CONCLUSION}

We successfully developed a noncontact method to predict the Hct of a DBS based on its total $\mathrm{Hb}$ content. In contrast to other published DBS-based total $\mathrm{Hb}$ analyses, this method is quantitative, nondestructive, does not require any sample preparation nor filter paper pretreatment, and most importantly, allows to predict the Hct of both fresh and old DBSs. This method was thoroughly validated and complied with all the predefined acceptance criteria generally used for bioanalytical procedures. Application on patient samples with a wide Hct range showed the applicability of this method on real patient samples. Although a slight bias could be observed, which could be attributed to (a combination of) several factors, this does not jeopardize the usefulness of the approach. This noncontact diffuse reflectance spectroscopy-based method overcomes the need for sample preparation, which in turn reduces the analysis time, minimizes the possibility of errors and, importantly, eliminates the need for sample destruction. Indeed, no part of the already limited sample volume of DBSs needs to be sacrificed for the additional Hct analysis, since mere scanning of a DBS suffices to derive the approximate Hct of the sample, one of the most important variables in DBS analysis. The predicted Hct could either be used to evaluate whether the Hct of a sample lies within the valid Hct range established during method validation or to correct the obtained result for the Hct effect anticipated at that Hct value. In both cases it is important to know the Hct of a DBS, even if the Hct value could also be determined on a corresponding liquid venous whole blood sample, as the venous and capillary Hct may not be the same. ${ }^{41}$ In addition, the capillary Hct also shows more variation during consecutive measurements than the venous 
Hct does, ${ }^{42}$ which may cause a larger variation in capillary analyte concentrations, as the latter are all subject to a (slightly) different Hct effect. Yet again, knowing the DBS's Hct may be beneficial in this context, as correcting the capillary DBS analyte concentration based on the predicted Hct of that exact DBS may help to reduce the variability. In addition, the predicted Hct may also be used to convert a DBS-based analyte concentration into the corresponding plasma-based concentration. Such a blood-plasma conversion is typically required to allow comparison of the DBS-based result with existing plasma-based reference values or therapeutic intervals and is often Hct-dependent, since the distribution of a compound between the red blood cell- and plasma fraction is often influenced by the amount of red blood cells present (i.e., a patient's Hct). Importantly, all the solutions which have hitherto been suggested to cope with the Hct effect on the spreading of the blood through the filter paper still yield a dried blood sample and therefore a dried blood sample-based result, necessitating a blood-plasma conversion and hence, a Hct determination in those cases as well. The only exception to this is the use of in situ generated DPSs. However, also this approach has its (Hct-related) issues. ${ }^{23}$

In future, the newly developed noncontact method will have to be applied on true capillary samples and it will need to be evaluated whether the predicted Hct will allow adequate Hct effect correction. Moreover, since the employed equipment is simple and compact, automation of this analysis can be easily envisaged.

\section{ASSOCIATED CONTENT}

\section{S Supporting Information}

The Supporting Information is available free of charge on the ACS Publications website at DOI: 10.1021/acs.analchem.6b01321.

Photograph of the utilized experimental set-up, flow chart of how the spectral data analysis is performed, overview of the Hct values of calibrators and QCs, equation used to calculate the normalized predicted Hct, choice of illuminated spot diameter in the context of DBS inhomogeneity and method reproducibility, influence of different anticoagulants on the reflectance spectra of DBSs, overview of the data for accuracy and precision for donor 2, comparison of predicted and true Hct values on $55 \mathrm{Li}$-heparin anticoagulated DBSs, influence of the $\mathrm{MCHC}$ value on the predicted Hct and influence of the hemolytic, lipemic, and icteric indexes on the predicted Hct. (PDF)

\section{AUTHOR INFORMATION}

\section{Corresponding Author}

*E-mail: christophe.stove@ugent.be. Tel.: +32 92648135 . Fax: +3292648183.

\section{Author Contributions}

${ }^{\S}$ Equally contributed

\section{Notes}

The authors declare no competing financial interest.

\section{ACKNOWLEDGMENTS}

The authors wish to acknowledge the skillful staff of the LAKC at the AMC, Amsterdam, and the Laboratory of Clinical Biology at Ghent University Hospital. Furthermore, they wish to thank the blood collection services of both hospitals, as well as the volunteers who participated in this study. SC. would also like to acknowledge the FWO Research Foundation-Flanders for granting her a $\mathrm{PhD}$ fellowship (application number $11 \mathrm{R} 7316 \mathrm{~N})$.

\section{REFERENCES}

(1) Velghe, S.; Capiau, S.; Stove, C. P. Trends in Analytical Chemistry 2016, DOI: $10.1016 /$ j.trac.2016.01.030.

(2) Therrell, B. L.; Padilla, C. D.; Loeber, J. G.; Kneisser, I.; Saadallah, A.; Borrajo, G. J. C.; Adams, J. Seminars in Perinatology 2015, 39, 171-187.

(3) Wilhelm, A. J.; den Burger, J. C. G.; Swart, E. L. Clin. Pharmacokinet. 2014, 53, 961-973.

(4) Edelbroek, P. M.; van der Heijden, J.; Stolk, L. M. L. Ther. Drug Monit. 2009, 31, 327-336.

(5) Stove, C. P.; Ingels, A.-S. M. E.; De Kesel, P. M. M.; Lambert, W. E. Crit. Rev. Toxicol. 2012, 42, 230-243.

(6) Sadones, N.; Capiau, S.; De Kesel, P. M. M.; Lambert, W. E.; Stove, C. P. Bioanalysis 2014, 6, 2211-2227.

(7) Ingels, A.-S. M. E.; Hertegonne, K. B.; Lambert, W. E.; Stove, C. P. CNS Drugs 2013, 27, 233-237.

(8) De Kesel, P. M. M.; Sadones, N.; Capiau, S.; Lambert, W. E.; Stove, C. P. Bioanalysis 2013, 5, 2023-2041.

(9) Abu-Rabie, P.; Denniff, P.; Spooner, N.; Chowdhry, B. Z.; Pullen, F. S. Anal. Chem. 2015, 87, 4996-5003.

(10) Li, F.; Zulkoski, J.; Fast, D.; Michael, S. Bioanalysis 2011, 3, 2321-2333.

(11) Youhnovski, N.; Bergeron, A.; Furtado, M.; Garofolo, F. Rapid Commun. Mass Spectrom. 2011, 25, 2951-2958.

(12) Meesters, R. J. W.; Zhang, J.; van Huizen, N. A.; Hooff, G. P.; Gruters, R. A.; Luider, T. M. Bioanalysis 2012, 4, 2027-2035.

(13) Lenk, G.; Sandkvist, S.; Pohanka, A.; Stemme, G.; Beck, O.; Roxhed, N. Bioanalysis 2015, 7, 2085-2094.

(14) Leuthold, L. A.; Heudi, O.; Deglon, J.; Raccuglia, M.; Augsburger, M.; Picard, F.; Kretz, O.; Thomas, A. Anal. Chem. 2015, 87, 2068-2071.

(15) Spooner, N.; Denniff, P.; Michielsen, L.; De Vries, R.; Ji, Q. C.; Arnold, M. E.; Woods, K.; Woolf, E. J.; Xu, Y.; Boutet, V.; Zane, P.; Kushon, S.; Rudge, J. B. Bioanalysis 2015, 7, 653-659.

(16) Denniff, P.; Spooner, N. Anal. Chem. 2014, 86, 8489-8495.

(17) Capiau, S.; Stove, V. V.; Lambert, W. E.; Stove, C. P. Anal. Chem. 2013, 85, 404-410.

(18) De Kesel, P. M. M.; Capiau, S.; Stove, V. V.; Lambert, W. E.; Stove, C. P. Anal. Bioanal. Chem. 2014, 406, 6749-6755.

(19) Mengerink, Y.; Mommers, J.; Qiu, J.; Mengerink, J.; Steijger, O.; Honing, M. Bioanalysis 2015, 7, 2095-2104.

(20) Kim, J.-H.; Woenker, T.; Adamec, J.; Regnier, F. E. Anal. Chem. 2013, 85, 11501-11508.

(21) Li, Y.; Henion, J.; Abbott, R.; Wang, P. Rapid Commun. Mass Spectrom. 2012, 26, 1208-1212.

(22) De Kesel, P. M. M.; Capiau, S.; Lambert, W. E.; Stove, C. P. Bioanalysis 2014, 6, 1871-1874.

(23) Sturm, R.; Henion, J.; Abbott, R.; Wang, P. Bioanalysis 2015, 7, 1987-2002.

(24) De Kesel, P. M. M.; Lambert, W. E.; Stove, C. P. Anal. Chim. Acta 2015, 881, 65-73.

(25) Bremmer, R. H.; Nadort, A.; van Leeuwen, T. G.; van Gemert, M. J. C.; Aalders, M. C. G. Forensic Sci. Int. 2011, 206, 166-171.

(26) Edelman, G.; van Leeuwen, T. G.; Aalders, M. C. G. Forensic Sci. Int. 2012, 223, 72-77.

(27) Sundharagiati, B.; Harinasuta, C. Trans. R. Soc. Trop. Med. Hyg. 1964, 58, 579.

(28) Feraudi, M.; Mejia, L. A. Am. J. Clin. Nutr. 1987, 45, 790-796.

(29) Kerti, A.; Morlin, Z. M.; Ferenc, P.; Laszlo, B. Animal Welfare, Ethology and and Housing Systems 2013, 9, 513-516.

(30) Orsini, J. J.; Yeman, J.; Caggana, M.; Bodamer, O. A.; Muehl, A. Clin. Chim. Acta 2010, 411, 894-895. 
(31) Yu, C.; Zhang, J.; Yuan, Z.; Liu, H.; Wang, X.; Wang, M.; Zou, L. Anal. Bioanal. Chem. 2015, 407, 8121-8127.

(32) Miller, J. H., IV; Poston, P. A.; Rutan, S. C.; Thomas, K. H. J. Anal. Bioanal. Tech. 2013, 4, 162.

(33) Cecchi, C.; Pieri, A.; Nassi, P.; Fanelli, A.; Liguri, G. European Journal of Clinical Chemistry and Clinical Biochemistry 1995, 33, 519524.

(34) Yang, X.; Piety, N. Z.; Vignes, S. M.; Benton, M. S.; Kanter, J.; Shevkoplyas, S. S. Clin. Chem. 2013, 59, 1506-1513.

(35) Edelman, J. G.; Roos, M.; Bolck, A.; Aalders, M. C. G. Journal of Selected Topics in Quantum Electronics 2016, DOI: 10.1109/ JSTQE.2016.2536655.

(36) Almeida, A. M.; Castel-Branco, M. M.; Falcao, A. C. J. Chromatogr. B: Anal. Technol. Biomed. Life Sci. 2002, 774, 215-222.

(37) U.S. Department of Health and Human Services, Food and Drug Administration, Center for Drug Evaluation and Research, Center for Veterinary Medicine. Draft Guidance for Industry. Bioanalytical Method Validation. http://www.fda.gov/downloads/ drugs/guidancecomplianceregulatoryinformation/guidances/ ucm368107.pdf (accessed April 2016).

(38) De Kesel, P. M. M.; Lambert, W. E.; Stove, C. P. Clin. Pharmacokinet. 2014, 53, 763-771.

(39) Sadones, N.; Archer, J. R. H.; Ingels, A.-S. M. E.; Dargan, P. I.; Wood, D. M.; Wood, M.; Neels, H.; Lambert, W. E.; Stove, C. P. Drug Test. Anal. 2015, 7, 336-340.

(40) Ingels, A.-S. M. E.; De Paepe, P.; Anseeuw, K.; Van Sassenbroeck, D.; Neels, H.; Lambert, W. E.; Stove, C. P. Bioanalysis 2011, 3, 2271-2281.

(41) Cable, R. G.; Steele, W. R.; Melmed, R. S.; Johnson, B.; Mast, A. E.; Carey, P. M.; Kiss, J. E.; Kleinman, S. H.; Wright, D. J. Transfusion 2012, 52, 1031-1040.

(42) Bond, M. M.; Richards-Kortum, R. R. Am. J. Clin. Pathol. 2015, $144,885-894$. 